Meta

Journal des traducteurs

Translators' Journal

\title{
Les sept principes cardinaux d'une didactique de la traduction
}

\section{Jean-Claude Gémar}

Volume 41, numéro 3, septembre 1996

URI : https://id.erudit.org/iderudit/002842ar

DOI : https://doi.org/10.7202/002842ar

Aller au sommaire du numéro

Éditeur(s)

Les Presses de l'Université de Montréal

ISSN

0026-0452 (imprimé)

1492-1421 (numérique)

Découvrir la revue

Citer cette note

Gémar, J.-C. (1996). Les sept principes cardinaux d'une didactique de la traduction. Meta, 41(3), 495-505. https://doi.org/10.7202/002842ar
Résumé de l'article

L'auteur établit une typologie des difficultés que rencontrent les professeurs de traduction dans l'établissement d'une méthode d'apprentissage répondant aux besoins des étudiants et aussi du marché du travail. Il présente sept grandes catégories qui touchent au cœur même du problème de la pédagogie de la traduction et de la réflexion méthodologique qui en découle. 


\section{LES SEPT PRINCIPES CARDINAUX D'UNE DIDACTIQUE DE LA TRADUCTION \\ Résumé}

L'auteur établit une typologie des difficultés que rencontrent les professeurs de traduction dans l'établissement d'une méthode d'apprentissage répondant aux besoins des étudiants et aussi du marché du travail. Il présente sept grandes catégories qui touchent au cœur même du problème de la pédagogie de la traduction et de la réflexion méthodologique qui en découle.

\section{Abstract}

The author classifies/categorizes the difficulties encountered by translation professors in developing teaching strategies adapted to both the needs of the student and market requirements. He then presents 7 broad categories vital to translation pedagogy followed by a few methodological considerations.

Si l'on s'interroge depuis toujours ou presque sur la manière de traduire, en revanche ce n'est que depuis quelques décennies que l'on se pose vraiment la question : comment enseigner à traduire?

D'ailleurs, avant même de poser cette questionlà, n'y aurait-il pas lieu de se demander si l'on peut enseigner à traduire?

Tel était bien le sens de l'exclamation que lançait, en 1957, Jean-Paul Vinayl. Or, ce qui va (presque) sans dire, aujourd'hui, était alors loin d'être le cas. À ce moment-là, la nécessité de former des personnes en vue d'exercer le métier de traducteur professionnel était loin d'être évidente. Signalons au passage que l'argument avancé par les négateurs de toute entreprise systématique de formation ne manquait pas de poids : la traduction étant (généralement considérée comme) un art, elle ne peut être enseignée. On n'enseigne pas le talent, encore moins le génie...

C'était oublier que l'activité traduisante ne se limite pas - ou ne se limite plus - aux seuls textes littéraires (ou «esthétiques»), loin de là. Elle porte sur de nombreux genres et domaines d'où toute considération autre que «pragmatique» (entendre: pratique) ou «communicative», selon les points de vue, est exclue. Et cela, sans doute depuis les origines de la traduction, soit depuis que les hommes ont arbitraire- ment décidé, un jour, que leur univers devait être scindé en deux, celui de la nature, d'une part, et celui de la culture, d'autre part. D'un côté, les arts et les lettres, de l'autre, les sciences. Aussi les préoccupations relatives à l'enseignement de la traduction reflètent-elles cet etat d'esprit. Traditionnellement, la traduction dite littéraire était l'apanage des universités et des programmes d'apprentissage des langues étrangères ou mortes; la traduction pratique (en gros : commerciale et technique) était enseignée dans des écoles spécialisées, des instituts, mais non à l'université, sinon exceptionnellement.

La pédagogie de la traduction s'en est longtemps ressentie, selon que l'enseignement était dispensé ici ou là. En outre, elle est restée fortement marquée par la tradition de l'enseignement des langues mortes (latin et grec), alors qu'elle portait, depuis longtemps déjà, aussi sur des langues vivantes, dites «modernes» par rapport aux «classiques». Quels qu'aient été les mérites - indéniables - de cette pédagogie classique, qu'on le veuille ou non son objectif était moins professionnel que linguistique : apprentissage, acquisition et perfectionnement des langues étrangères, notamment en vue de les enseigner. Son objet enfin, procédant d'une tradition humaniste à laquelle l'université reste très attachée, était avant tout culturel.

Le fossé entre la tradition obligée du passé aussi brillant fût-il — et la nécessité imposée par le présent s'est lentement creusé dans la première moitié du XXe siècle. La Deuxième Guerre mondiale, en l'occurrence, a joué un rôle de révélateur et, surtout, d'accélérateur. Les mouvements de troupes à travers le monde, de l'Europe au Japon, ont imposé l'évidence, à savoir que l'enseignement traditionnel des langues, notamment des langues vivantes, n'était plus adapté à la situation. À situation nouvelle, pédagogie nouvelle. Les Américains, dont les Gls puis les hommes d'affaires furent tour à tour confrontés à des situations linguistiques auxquelles ils étaient peu ou mal préparés, surent s'adapter aux circonstances et trouver les formules pédagogiques appropriées, c'est-à-dire simples mais efficaces. L'enseignement de la traduction en a été profondément modifié, et le hiatus entre l'apprentissage de la traduction par l'enseignement des langues et l'apprentissage de la traduction per se n'a cessé de s'accentuer. Il demeure au cceur du problème que pose la formation des traducteurs. En gros, le professeur de traduction est appelé à choisir entre la «stylistique comparée», façon Vinay et Darbelnet et ses variantes, et les nombreuses formules à vocation multiple - dont professionnelle - meublant le marché actuel de la formation du traducteur.

La création, dans les années 50 , des premières écoles universitaires de traduction, en Europe et au Canada, n'a pas pour autant réglé la question de la formule pédagogique souhaitable. La pédagogie de la traduction est, aujourd'hui encore, fortement imprégnée de classicisme et de traditionalisme dans de nombreux pays. En 1976 encore, la Conférence générale de l'Unesco, dite conférence de Nairobi, 
formulait, à l'article 11 de sa recommandation, la déclaration suivante:

Les Etats membres devraient reconnaître le principe selon lequel la traduction est une discipline autonome dont l'enseignement doit être distinct de l'enseignement exclusivement linguistique et qui requiert une formation spécialisée.

Si la traduction est bien une discipline autonome et si son enseignement doit être dissocié de l'enseignement traditionnel exclusivement linguistique, il faut alors concevoir un type de formation particulière, voire spécialisée reposant sur une méthodologie appropriée, qu'elle vise à former des traducteurs en général (des «généralistes» de la traduction, en somme) ou des «spécialistes», selon les besoins du marché local, régional, national ou encore international.

Cela suppose également que l'on ait sinon résolu tous les problèmes de pédagogie que ce choix entraîne, du moins réfléchi à ses éventuelles conséquences. Autrement dit, pour reprendre un mot de Jean Darbelnet, il s'agit de concilier «la façon dont on conçoit la traduction et la façon dont on l'enseigne ${ }^{2} \gg$. $\mathrm{Ce}$ qui présuppose que le professeur de traduction, comme tout professeur digne de ce nom, aura réfléchi un tant soit peu à son enseignement et, sans aller jusqu'à devoir élaborer un système théorique, sa propre théorie de la traduction, possédera «au moins un embryon de théorie sur la matière qu'il enseigne ${ }^{3}$ ». Par exemple, entre autres questions fondamentales que l'on devrait se poser et auxquelles on se doit de répondre, est-il préférable d'enseigner à traduire vers sa langue plutôt que vers la langue étrangère? Ou dans les deux sens? Ou encore, doit-on opter pour une manière plutôt que pour une autre, par exemple prôner la traduction du sens, la traduction idiomatique, ou, au contraire, former à traduire littéralement, mot à mot? On sait que l'une et l'autre comptent de nombreux adeptes.

Les réponses à des questions de cette nature ne nous appartiennent qu'en partie. Elles nous sont imposées, en réalité, par les contraintes (sociales, linguistiques, économiques, politiques, culturelles, etc.) de la société dans laquelle nous vivons. Selon que le contexte local sera unilingue, bilingue ou multilingue, par exemple, les contraintes et les risques découlant des choix envisagés posent un sérieux dilemme à tout enseignant appelé à ouvvrer dans un tel contexte. Ils constituent des enjeux pédagogiques fondamentaux parce qu'ils peuvent hypothéquer l'avenir, professionnel comme personnel, des élèves ou des étudiants.

Ces enjeux seront différents de ceux auxquels la grande majorité des professeurs ont à faire face, qui enseignent généralement en milieu essentiellement unilingue. En effet, les références culturelles des élèves comme des professeurs participent de la même culture. En revanche, les enjeux de l'enseignement de la traduction vers le français en milieu bilingue et, pour ce qui me concerne, au Québec, s'adressent plutôt à la conscience de l'enseignant, placé devant ses responsabilités (morales, sociales, culturelles, et même politiques) vis-à-vis de ses élèves.

Sur le plan moral tout d'abord, celui du genre d'apprentissage qu'il convient de donner à l'élève, doit-on former un généraliste, un spécialiste ou, plus simplement, un être humain, une conscience? C'est l'alternative fondamentale entre le professionnalisme, la spécialisation, et l'universalisme de la formation.

Sur les plans social et culturel, ces enjeux engagent l'avenir de l'élève, son entrée plus ou moins facile sur le marché du travail, son intégration plus ou moins réussie à la société dans laquelle il est appelé à vivre comme membre d'un «groupe ethnique ${ }^{4}$ » concourant à la réalisation d'un mode de vie commun.

Sur le plan politique enfin, entendu au sens noble du terme, l'enseignant est placé devant un dilemme d'une autre sorte, selon le type de critères ou de normes qu'il retiendra, il fera un choix de société, car l'idéologie sous-tend alors sa décision. D'un côté, opter pour la «pureté d'origine» de la langue et de la culture équivaut à accentuer le caractère unique, pur et dur, d'une société généralement peu portée au compromis linguistique, car la langue est une des «cordes sensibles" qui vibre au moindre frottement ${ }^{5}$. De l'autre, accepter la nature mixte d'une culture - la canadienne, par exemple - et son langage revient à jouer le jeu institutionnel selon les règles fixées par le législateur (fédéral) et le bilinguisme officiel.

Comme on voit, ces enjeux dépassent de très loin les responsabilités pédagogiques normales du professeur de langues ou de traduction. Ils ne concernent qu'en faible partie la formation des individus et des esprits et touchent véritablement à l'essence de l'être, à ses options fondamentales et, de ce fait, engagent moralement l'enseignant. Celui-ci est alors tenu non seulement quant aux résultats, mais encore quant aux moyens de les atteindre.

Cela dit, la plupart des spécialistes de la traduction, les «pédagotrads», s'ils arrivent difficilement à s'entendre sur ce que devrait être la méthode idéale d'enseignement de la traduction, s'entendent généralement sur ce que devrait posséder le traducteur pour exercer son activité dans les meilleures conditions une connaissance indispensable des langues (de départ et d'arrivée) et une culture générale suffisante. En théorie, tout le dilemme de la formation du traducteur se résume à deux grandes catégories de problèmes :

\section{Compréhension (de la langue/du texte de départ, LD/TD \\ 2. Réexpression (dans la langue/le texte d'arrivée, LA/TA)}

Tout découle de ce postulat de base, la culture générale comme la connaissance des langues, la compétence du traducteur et sa performance. La didactique de la traduction doit nécessairement emprunter ce passage obligé. 
Sans prétendre vouloir établir une typologie exhaustive ${ }^{6}$ des difficultés que rencontre le professeur de traduction dans l'établissement d'une méthode d'apprentissage répondant aux besoins de ses étudiants, j'ai recensé sept grandes catégories de problèmes généraux qui, tout en se posant particulièrement dans le contexte canadien?, me paraissent néanmoins suffisamment exemplaires pour leur voir reconnaitre un caractère universel. Ils ressortent, en effet, assez nettement des programmes de formation de traducteurs de quelque 20 régions et pays du monde entier rassemblés dans une étude publiée par l'AUPELF, en 1988, sous ma direction ${ }^{8}$. En voici la liste:

1. méthodes d'enseignement de la traduction;

2. langue(s) de départ (LD);

3. langue d'arrivée (LA);

4. culture générale;

5. documentation et terminologie;

6. évaluation d'une traduction;

7. pratique de la traduction.

Je vais aborder ces différentes questions, sans trop entrer dans les détails de chacune d'elles, ce qui pourrait faire l'objet d'une, et même de plusieurs thèses de doctorat tellement elles touchent au coeur du problème de la pédagogie de la traduction et de la réflexion méthodologique qui en découle.

\section{LES MÉTHODES D'ENSEIGNEMENT DE LA TRADUCTION}

Vinay et Darbelnet, parmi les premiers, ont convaincu le monde universitaire de la nécessité d'enseigner la traduction selon une méthode rigoureuse, mieux adaptée à la société et à ses besoins actuels, et fondée sur des principes qui, quoique inspirés par la linguistique, sont applicables à l'enseignement des langues vivantes dans une perspective de traduction. Qui ne connait les sept procédés ${ }^{9}$ de traduction exposés dans la Stylistique comparée du français et de l'anglais, célèbres dans toutes les écoles de traduction, aux quatre coins du monde, quelles que soient les langues que l'on $y$ enseigne?

Depuis sa parution, cet ouvrage a imposé un style sinon une «méthode», terme que les auteurs mêmes ont longtemps contesté ${ }^{10}$, lui préférant celui de "procédés». Ses mérites sont trop connus pour être repris encore une fois ici. Par la suite, nombre d'ouvrages ont paru - dans différentes langues sur le sujet, notamment dans la ligne de Vinay et Darbelnet. D'autres écoles ont cherché leur propre voie et un mode d'expression original, souvent 'par opposition' au «Vinay-Darbelnet» ou par-devers lui. D'autres courants enfin, chacun d'eux se fondant sur un champ particulier de la connaissance, ont apporté leur contribution à la théorie générale de la traduction et à sa pédagogie.

L'ouvrage de référence qu'est la Stylistique comparée, quoiqu'il reste une des bibles du traducteur, a néanmoins vieilli. N'oublions pas qu'il a été pensé au début des années 1950 par des linguistes formés dans les années 1920 et 1930 . On lui a notamment reproché de n'être qu'une version modernisée des méthodes d'enseignement des langues, ce qui est en partie vrai, et d'avoir puisé son inspiration stylistique chez Charles Bally, ce qui est indéniable: les maîtres ont, de tout temps, inspiré leurs disciples! On sait ce que doit la linguistique moderne à Ferdinand de Saussure, et l'actuelle, aux travaux de Noam Chomsky. On a surtout reproché à Vinay et Darbelnet de proposer une méthode d'enseignement $a$ posteriori de la traduction, une fois les difficultés résolues et la solution trouvée, ce qui est également vrai. Mais ce reproche peut être étendu à la plupart des méthodes en cours, y compris à celles des détracteurs les plus virulents de Vinay et Darbelnet, comme le démontre éloquemment Daniel Gouadec dans un article paru dans Meta $^{11}$, où il renvoie dos à dos les principaux protagonistes, parce que «sous la diversité des emballages se cache une identité de principes ${ }^{12}{ }_{\text {». }}$. Si, désormais, on ne peut plus mettre en doute le fait que la traduction peut être enseignée, il est toujours permis de se demander : comment?

Malgré le prestige de méthodes comme celle que Vinay et Darbelnet ont conçue, force est de reconnaitre que l'enseignement de la traduction, mis à part quelques exceptions et progrès notables ${ }^{13}$, a relativement peu évolué par rapport à celui d'autres disciplines et que, dans de nombreux pays, il s'inspire encore de méthodes archaïques et, dans bien des cas, dépassées. Cette pédagogie sommaire ne saurait suffire à former les traducteurs professionnels dont la société actuelle a besoin, notamment à l'échelle des organisations internationales. Par ailleurs, le décalage entre le monde de la formation et le monde du travail reste grand. Les employeurs reprochent souvent - et parfois à tort - aux universités de ne pas avoir adapté leur enseignement aux besoins «réels» de la profession ${ }^{14}$.

Il convient de reconnaître que certaines écoles et traditions ${ }^{15}$ font une part encore trop belle à la formation théorique, au détriment de la pratique. Au contraire, d'autres insistent trop sur le côté pratique et négligent les connaissances générales, qui sont pourtant une des composantes essentielles de la formation du traducteur. La difficulté, là comme ailleurs, consiste à trouver un équilibre souhaitable entre théorie et pratique. Il s'ensuit que la traduction est une discipline qui est encore en quête d'une méthodologie ${ }^{16}$, situation qu'elle doit au retard qu'ont pris les sciences humaines sur les sciences exactes. Le mélange incertain d'empirisme mal intégré et de théorie floue mal digérée caractérisant l'enseignement actuel de la traduction professionnelle s'inspire encore trop peu des progrès de la linguistique générale comme des sciences, entre autres sociales.

Enfin, sauf dans quelques pays privilégiés, le manque de maîtres qualifiés pour enseigner la traduction oblige les responsables des programmes à recourir aux services de praticiens formés sur le tas. 
Ces derniers, traducteurs compétents, n'ont en général pas été formés aux méthodes pédagogiques convenant à une situation d'apprentissage en milieu universitaire et, qui plus est, n'ont pas tous reçu une formation universitaire eux-mêmes ${ }^{17}$.

Tout cela concourt à poser le problème général des méthodes d'enseignement de la traduction, des choix à faire en fonction de telle situation, et à s'interroger sur les fondements pédagogiques d'une discipline qui, contrairement à nombre d'autres ${ }^{18}$, est toujours en quête d'une méthodologie.

\section{LA LANGUE DE DÉPART (LD)}

Une plainte générale s'élève de toutes parts chez les professeurs de traduction, et cela dans tous les pays : les étudiants, durant leur apprentissage et même à la sortie des écoles de formation, ne possèdent pas la langue de départ au niveau attendu d'un traducteur. En conséquence, comprenant mal certaines parties du texte à traduire, ils produiront une traduction d'une qualité douteuse ${ }^{19}$. De là un nombre élevé de fautes de traduction dues à la méconnaissance de LD. Les employeurs sont formels ${ }^{20}$, à commencer par le premier d'entre eux (l'État canadien), il s'agit d'une des lacunes les plus graves que l'on relève dans la formation du traducteur, et que l'on impute habituellement aux formateurs. En sont-ils vraiment responsables?

Tout d'abord, il y a la situation linguistique du pays considéré. Selon que l'on aura affaire à un pays unilingue (Brésil, par exemple), bilingue (Belgique) ou multilingue (Suisse), l'accent sera mis sur $L D$ ou LA. Ensuite, l'apprentissage visera l'acquisition passive ou active de LD en fonction des orientations spécifiques du programme, des besoins des étudiants (bilinguisme actif ou passif, unilinguisme). La formation d'étudiants déjà bilingues lorsqu'ils entrent dans une école de traducteurs, qu'il s'agisse d'un premier ou d'un second cycle ${ }^{21}$, suivra un cheminement fort différent de celle d'étudiants n'ayant reçu qu'une formation sommaire en LD. Ces derniers devront alors acquérir une solide formation en $\mathrm{LD}$.

Dans la première hypothèse, $s$ 'il est possible de décharger les étudiants bilingues de certains cours de langue au profit de cours extra-linguistiques (culture et connaissances générales, par exemple), le phénomène des interférences (de LD vers LA, en général), qui affaiblissent l'usage de la langue maternelle ou vernaculaire, ne doit pas être ignoré. Il importe alors de renforcer celle-ci par des moyens pédagogiques appropriés. Dans la seconde, celle d'étudiants unilingues possédant des notions de la langue seconde, il s'agira d'amener ces derniers au niveau de connaissance nécessaire mais suffisant de LD pour traduire efficacement. Il faudra alors prévoir une progression calculée selon des objectifs précis et en fonction des buts du programme, et non chercher à atteindre l'équilibre de la compétence dans les deux langues, qui est le propre des bilingues.

Dans les deux cas, l'apprentissage d'une troisième langue, d'une quatrième, etc. se posera en termes à peu près identiques, l'objectif premier étant d'arriver à une connaissance fonctionnelle de la ou des langues considérées. Pour ce faire, la situation linguistique particulière de tel pays (ou région) conditionnera les moyens à mettre en œuvre pour l'apprentissage d'une langue seconde, troisième, quatrième, etc.

De LD à LA; il n'y a qu'un pas à franchir, celui, justement, que l'opération traduisante est censée rendre possible.

\section{LA LANGUE D'ARRIVÉE (LA)}

On s'étonnera peut-être de trouver la langue d'arrivée citée parmi les problèmes méthodologiques que pose l'enseignement de la traduction. En effet, la langue d'arrivée étant le plus souvent la langue maternelle du traducteur, on se demande où est le problème. Il est plus grave qu'on ne l'imagine. Il n'est pas entièrement imputable aux étudiants, contrairement à ce que certains voudraient laisser croire ${ }^{22}$, mais en grande partie à la société, à son évolution depuis la Deuxième Guerre mondiale et au culte de l'oral, de l'audiovisuel, de l'image, le tout au détriment de l'écrit, de la maîtrise de la syntaxe et des règles de la grammaire (déclin des langues mortes classiques), de l'orthographe et, pour finir, de la rédaction, qui n'en finissent pas de se détériorer.

$\mathrm{Ce}$ phénomène est universel, comme nous l'avons amplement constaté dans de nombreux pays. Ce mal frappe tout le monde, le développé comme l'autre: le tiers monde, les pays riches (Canada, ÉtatsUnis, France et même Japon !) comme les pays en développement (Brésil, Colombie, Mexique, Venezuela, etc.), les pays de l'Europe de $1^{\prime} E s t$ comme ceux d'Asie.

Toutes proportions gardées, le fait que l'ingénieur, le médecin ou l'avocat actuel possède moins bien qu'autrefois les fondements de sa langue est peut-être (relativement) moins grave que de faire ce constat pour ceux qui sont véritablement des professionnels de la langue: interprètes, traducteurs, terminologues, rédacteurs et réviseurs.

Toute traduction, qu'elle soit littérale (mot à mot), qu'elle privilégie le sens par rapport à la forme ou qu'elle recoure à l'adaptation (par exemple: thème à thème), fait intervenir, à un degré quelconque, la rédaction. Que le traducteur, au sommet de son art, tende vers la création (littéraire) ou qu'il vise uniquement à composer un texte destiné à la communication pratique (technique, commercial), il rédige. Pour cela, il doit posséder les règles élémentaires de cette technique, voire les maîtriser. Aussi, devant les lacunes constatées chez l'étudiant, dans la plupart des écoles de formation de traducteurs, a-t-on dû intégrer au programme des cours de rédaction adaptés aux besoins particuliers des étudiants, qui ne sont pas les mêmes à Caracas qu'à Mexico, à New York qu'à Toronto, à Montréal qu'à Paris, Genève ou Mons. En fait, le véritable problème est celui de la connaissance, plus ou moins poussée selon la nature du texte 
et son contenu, de la ou des (cas des bilingues) langues matemelles, indispensables pour traduire. Une nouvelle contrainte pédagogique est donc venue s'ajouter à celles qui pèsent déjà sur le professeur de traduction, soit de devoir renforcer, consolider les connaissances de la langue maternelle, par voie de cours de grammaire, de stylistique, de rédaction. Ses obligations linguistiques, en tant que pédagogue, sont désormais doubles, elles s'exercent vis-à-vis de LD ('compréhension' plus ou moins profonde du TD) et de LA ('réexpression' du sens/message dans le TA sous une forme 'satisfaisante').

Les liens entre langue et culture (générale) sont étroits. La question de la culture du traducteur pose de nombreuses difficultés méthodologiques, donc pédagogiques.

\section{LA CULTURE DU TRADUCTEUR}

Le troisième motif de doléance des employeurs, comme des professeurs également, porte sur le manque de culture «générale» des traducteurs à la sortie de l'université. Nous ne chercherons pas à en analyser les causes, qui procèdent probablement des mêmes faits de société que ceux qui caractérisent les maux dont souffre la langue en général. Nous nous attacherons plutôt, à partir de ce constat, à envisager des solutions méthodologiques propres à corriger la situation, en vue de proposer au traducteur des moyens d'acquérir par lui-même le minimum de connaissances indispensables pour exercer un métier très exigeant sur ce plan-la.

On entend souvent dire, selon la formule bien connue, que «la culture générale, c'est ce qui reste quand on a tout oublié». Dans le courant actuel de spécialisation à outrance, ce «reste» fond comme neige au soleil au bénéfice des connaissances spécialisées. Le recul des études classiques et littéraires, l'absence d'intérêt pour l'histoire et la philosophie, le désintérêt pour la géographie - physique, économique et humaine - expliquent en partie le déclin (relatif) de la culture dite générale de l'étudiant lorsqu'il accède à l'université. Alors que la fonction première du traducteur consiste à faire passer le message contenu dans le TD, ce message, à défaut d'un niveau suffisant de culture générale, risque d'être, au mieux, tronqué et, à la limite, totalement faussé lorsque les allusions et les nuances culturelles n'ont été saisies qu'en partie voire pas du tout. C'est dire la place qui devtait être réservée à l'acquisition de cette culture dans la formation du traducteur. Mais avant de poursuivre, il s'agit de s'entendre sur une définition de cette notion de «culture générale». Celle que propose le Grand Larousse de la langue française nous semble répondre particulièrement à ce que l'on attend du traducteur:

Ensemble de connaissances, appartenant à tous les domaines de la pensée, considérées comme nécessaires avant toute spécialisation. (Nous soulignons.)
La teneur de la dernière proposition est frappante, notamment l'adverbe avant. Il s'ensuit qu'il est illusoire, surtout dans le cas d'un traducteur, de vouloir former des spécialistes qui seraient dépourvus des connaissances requises pour traduire des textes appartenant à un domaine différent de celui de la spécialité visée. De plus, très rares sont les textes spécialisés au point que le traducteur n'aura pas affaire à un autre champ de la pensee. Cela nous amène à préciser notre pensée sur le contenu de cette culture générale. On peut débattre sans fin du caractère "général» d'un texte. Pour certains, tel texte devrait être rangé dans la catégorie technique; pour d'autres, ce peut être scientifique; d'autres enfin y verraient un texte spécialisé. D'une certaine façon, le même texte pourrait être tout cela à la fois, selon que le lecteur sera un profane, un praticien ou un théoricien. Là, peut-être, réside le fonds de culture générale que doit posséder tout traducteur: il doit réunir les trois personnes en une.

\section{LE TRADUCTEUR, «HONNÊTE HOMME» DE SON TEMPS}

Les fondements de la culture traditionnelle ont été profondément ébranlés par les transformations subies par la société depuis une génération. Il semble que nous nous acheminions vers une forme de culture nouvelle, encore à l'état d'esquisse mais néanmoins sensible. D'ores et déjà, la culture, qui est, nous le rappelons, «la manière dont une collectivité construit ses relations avec son environnement) (Alain Touraine), ne repose plus sur le seul pilier de la littérature et de l'humanisme.

Aussi, le «patrimoine informationnel» de l'honnête homme du XXe siècle et, a fortiori du XXIe, ne peut-il plus être constitué de la seule connaissance des œuvres littéraires, du passé comme du présent. Il doit comprendre aussi, sinon davantage, la connaissance des faits, événements sociaux, économiques, politiques, scientifiques et techniques de notre temps. C'est ainsi que se forme une culture «générale». Elle doit être composée autant des savoirs que des savoirfaire d'une collectivité. Le traducteur, plus que tout autre artisan des faits de culture, étant donné son rôle de médiateur entre les langues, et donc les cultures, doit posséder les outils conceptuels lui permettant de mettre la langue pleinement au service de la société.

Le professeur de traduction doit prendre en compte cette réalité, la traduire en actes pédagogiques et mettre au point des formules visant à l'acquisition et au développement des fondements de cette nouvelle culture. Cela pourrait éviter que l'on trouve dans des copies d'examen, des fautes de traduction qui démontrent que la formation des étudiants souffre de lacunes graves sur le plan des connaissances générales. Par exemple, que l'on confonde Whitehall et la Maison-Blanche (White house), Commonwealth et Marché commun; que l'on Ecrive sans sourciller qu'en Angleterre, au XVIIle siècle, s'était établie une «république fédérale» (!); que la «Maison des 
Seigneurs» soit avancée comme traduction de House of Lords (Chambre des Lords), etc. ${ }^{23}$

Un autre problème méthodologique que doit résoudre le professeur de traduction est posé par la documentation et la terminologie.

\section{LA DOCUMENTATION ET LA TERMINOLOGIE}

Le problème de la documentation, en traduction, découle de la prolifération du vocabulaire, en expansion constante, due aux progrès accomplis par la science et les techniques. Les ouvrages de référence ont du mal à suivre une évolution aussi rapide. Aussi le professeur est-il placé devant un problème double.

D'une part, disposer du matériel pédagogique approprié utilisable en classe, auquel il renverra les étudiants pour qu'ils préparent les travaux à faire et les leçons à venir. Pour cela, il doit amasser une documentation plus ou moins exhaustive sur un sujet ou dans un domaine donné (technique, médical, juridique,...), l'évaluer, l'analyser et, le cas échéant, en faire une synthèse pour la présenter en classe. D'autre part, il doit préparer ses étudiants à la recherche documentaire en leur indiquant les ouvrages de référence à consulter pour effectuer leurs recherches - notionnelles ou terminologiques - et les orienter à l'aide d'un certain nombre de clés propres au domaine étudié.

A cela vient s'ajouter la difficulté proprement terminologique que présente la masse du vocabulaire, l'instabilité sémantique des néologismes qui abondent autant dans la langue courante que dans les langues techniques. Il s'agit alors de résoudre les problèmes de polysémie, synonymie, néologie, emprunts et calques que pose la traduction technique et scientifique de pointe. L'établissement de critères destinés à guider le choix du futur traducteur en fonction de normes prétablies est une des tâches les plus délicates du professeur de traduction, la norme étant, comme l'on sait, difficile à cerner et plus encore à saisir ${ }^{24}$. La norme est par definition arbitraire. En traduction, toutefois, elle doit guider le choix de la langue à enseigner, le critère étant en l'occurrence le destinataire du texte, le public-cible, en contexte de traduction professionnelle et spécialisée. Cette distinction se conçoit aisément. Un Anglais ne réagira pas aux mêmes allusions (stimuli) qu'un Américain; un New-Yorkais réagira différemment d'un habitant de San Francisco, de Chicago ou d'Atlanta; un Espagnol ou un Belge n'appréciera pas de la même manière un trait culturel qu'un Chilien ou un Québécois. Traduire un texte, c'est aussi en faire l'adaptation aux réalités culturelles, sociales et ethniques du public auquel il est destiné.

\section{DOCUMENTATION GÉNÉRALE}

\section{ET DOCUMENTATION SPÉCIALISÉE}

La recherche étant une opération consubstantielle à la traduction, quelles que soient les LD et LA, le traducteur commencera par chercher dans tel ouvrage la réponse à sa question et, à la suite d'une réflexion personnelle, l'adaptera aux conditions particulières du texte qu'il traduit : niveau de langue, tonalité, micro et macrostructure, destinataire, etc.

L'ennui, c'est que cette documentation répond rarement à son attente. Tel est notamment le cas lorsque le document a été établi dans un autre pays, conçu selon des schémas culturels différents de ceux du pays utilisateur, donc adapté à un contexte sociolinguistique, sociopolitique et socioéconomique parfois fort différent ${ }^{25}$. Ce problème est général et touche tous les pays partageant la même langue, sinon la même culture et les mêmes horizons. Entre pays francophones, par exemple, les références (climatiques, géographiques, sociales, politiques, culturelles, etc.) sont souvent «hexagonales». Elles n'ont que peu à voir avec les équivalents locaux. Si l'on prend la traduction française de la Bible comme exemple, que peut-elle évoquer pour un lecteur du sud du Cameroun? Que représente pour un Camerounais le «désert» biblique? L'olivier? Les chameaux et les tentes des bédouins? Ils ne font pas partie de leur univers naturel, la «neige» encore moins. La traduction de la Bible en boulou présente, à cet égard, des difficultés dont le traducteur ordinaire $n^{\prime a} a$ aucune idée.

Ensuite, le deuxième genre de problèmes que pose la documentation, les dictionnaires (et lexiques, glossaires, vocabulaires, thésaurus, etc.) notamment, découle des méthodes lexicographiques ou terminographiques mêmes. L'activité traduisante passe obligatoirement par la fréquentation des dictionnaires généraux et spécialisés. Lorsque Ic traducteur cherche une réponse dans un bon dictionnaire unilingue (OED, Webster ou Random House; Littré, Robert ou Larousse), il $y$ trouvera généralement ce qu'il cherche. On ne peut en dire autant des dictionnaires bilingues ou multilingues. Comment, en effet, un dictionnaire bilingue - et, a fortiori, multilingue pourrait-il proposer l'équivalent des grandes productions lexicographiques nationales que sont les monuments de la langue anglaise, française, allemande ou espagnole? Les Harrap's et autres Robert et Collins ne peuvent avancer, au mieux, que quelques milliers de mots par comparaison aux gigantesques corpus des derniers produits de la lexicographie moderne 26 . Contrairement aux langues mortes, dont le lexique est «fini», les langues vivantes produisent constamment du vocabulaire, des sens nouveaux, en perdent d'autres. Seul le cerveau humain, avec ses formidables possibilités, peut saisir les nuances des pièges que pose constamment la polysémie au traducteur. En anglais, le mot case compte, selon le Webster (édition de 1976), jusqu'à 34 acceptions; la préposition of, à elle seule, en comprend 29. Le mot français «pièce», dans le GLLF, a 23 acceptions, «coup» frôle les 20 . Et ce phénomène caractérise toutes les langues romanes et saxones. Le Grijalbo, en espagnol, dénombre quelque dix acceptions de mariposa, et l'on s'étonnera peut-être de voir le nombre d'acceptions 
d'un mot aussi «élémentaire» que pie, puerta, ou l'allemand sprechen, Sprache ou lustig.

Les limites de la lexicographie ressortent ici, malgré les tentatives des sémanticiens de faire le tour de la question du sens ${ }^{27}$. La difficulté n'est pas moindre dans les champs de spécialité, encore que certains domaines des sciences dites exactes (mathématiques, physique, chimie) possèdent une nomenclature sémantiquement stable et relativement bien définie. En sciences sociales et humaines, en revanche, on sait de quoi il en retoume. Les sciences juridiques et politiques, par exemple, ont une terminologie difficilement exportable, parce qu'elle s'identifie à une situation propre à un pays. Et quand bien même les institutions politiques ou les systèmes juridiques seraient (apparemment) semblables, les correspondances ne sont jamais parfaites. Peut-on comparer la Cour suprême des États-Unis à la Cour de cassation (ou au Conseil d'État, à la Cour des comptes), et cette dernière à la Chambre des Lords d'Angleterre? Non, bien sûr, et cela dans les deux sens.

Ces particularismes expliquent en partie pourquoi les bons dictionnaires de traduction sont aussi rares. En outre, plus le nombre de langues proposé en équivalent augmente, et plus la fiabilité de la réponse diminue... Aussi est-il préférable, dans la plupart des cas, s'il faut absolument recourir au dictionnaire pour comprendre le sens approximatif d'un mot, de s'en tenir aux dictionnaires unilingues et de ne consulter les ouvrages de traduction qu'en dernier recours. Les niveaux de discours caractérisant une langue donnée (courant, familier, technique, soutenu, administratif, etc.) sont le produit unique de facteurs spécifiques, et comme tels, difficilement transposables.

De l'utilisation bien comprise des ouvrages de référence à l'évaluation d'une traduction, il $\mathrm{y}$ a parfois un lien de cause à effet.

\section{ÉVALUATION D'UNE TRADUCTION}

Selon les conceptions du moment, les critères d'évaluation d'une traduction ont considérablement varié au cours des âges. De tout temps, depuis Cicéron au moins, les tenants de la traduction littérale se sont opposés à ceux de la traduction du sens. La dispute, quasi théologique, se poursuit de nos jours encore. Les critères d'une «bonne» traduction sont aussi nombreux et variés que les théories de la traduction. Encore s'agit-il de s'entendre sur ce que l'on qualifie de «faute» ou d'erreur et sur la conduite à suivre: sanctionner ou non. On sait que deux écoles de pensée, diamétralement opposées, se partagent la doctrine en la matière. Selon la conception américaine de la pédagogie, l'erreur étant la préfiguration d'un échec futur, elle doit être minimisée le plus possible : il faut insister sur le côté positif des choses et gommer le risque d'echec, traumatisant pour l'esprit. À l'inverse, selon la conception française (que partagent les Japonais), l'erreur révèle les lacunes d'une formation, qui doivent être comblées. Selon que l'on suivra l'un ou l'autre modèle, l'évaluation d'une traduction s'en ressentira.

Pourtant, les traducteurs s'entendent généralement sur ce que doit être une traduction: fidèle. Mais fidèle à quoi : à la lettre ou à l'esprit? Et une fois que l'on s'est entendu sur ce principe manichéen, son application relève d'une forme d'éthique professionnelle et, a la limite, de la morale philosophique: le droit à l'erreur; le libre arbitre; les niveaux possibles d'interprétation d'un texte; la lecture, et même les lectures différentes qu'en fera chaque personne; etc. Ensuite, l'erreur d'interprétation du sens que peut commettre une personne conditionne-t-elle le sort du texte traduit? Les erreurs que l'on prête aux traductions que Baudelaire et Mallarmé ont faites de l'cuvre d'Edgar Allan Poe portent-elles à conséquence? Le plaisir de lecture que nous procure la traduction de Beaudelaire en est-il altéré? Qu'en esti1, en revanche, dans le cas de la traduction d'une police d'assurance, si une erreur se glisse dans la formulation d'une clause de responsabilité ? Quelle en sera l'incidence sur son interprétation devant les tribunaux?

On le voit, les enjeux et la gravité d'une erreur, d'une «faute» de traduction n'ont pas le même sens ni la même portée selon que le texte aura vocation littéraire, ludique ou esthétique, ou, au contraire, portera sur un domaine ou une question ayant des conséquences fâcheuses pour l'intéressé. Peu importe, au fond, que Baudelaire ait parfois trahi ou déformé le texte de Poe; seul compte le plaisir évoqué plus haut. En revanche, si le pilote d'un avion pousse la manette $X$ ou $Y$ au lieu de la tirer vers lui, la fausse manouvre aura peut-être des conséquences très graves. Les mots et leur sens ne pèsent pas le même poids dans le premier et dans le deuxième cas. D'ailleurs, qu'entend-on par «faute»? Un «écart» par rapport à une norme? Or, ce qui, pour l'un, constitue une faute, pour l'autre passe peut-être pour juste, satisfaisant ou original. Les avis sont très partagés sur la question et les spécialistes ne s'entendent guère sur ce point ${ }^{28}$. Comme souvent, et comme en droit, deux conceptions s'opposent: l'interprétation stricte et l'interprétation large.

Une chose paraît sûre, en tout cas: la même «faute», selon que le texte sera lu (et éventuellement évalué, analysé et critiqué) par un profane, un spécialiste du domaine ou un expert en la matière, sera jugée et appréciée différemment. Nous sommes ici dans l'ordre du subjectif. La valeur de la faute est fonction de l'application, de l'usage qui sera fait du texte, de sa destination. La quête de l'objectivité dans l'évaluation de la qualité d'une traduction ne cesse de préoccuper les traducteurs ${ }^{29}$. Évaluer, c'est juger. Comment peut-on juger le plus objectivement possible le travail d'une personne si l'on ne s'appuie pas sur un certain nombre de critères reconnus? Or, ceuxci ont évolué et changé au fil du temps selon les façons de voir propres à chaque époque. Ils dépendent des buts visés par l'activité et l'opération traduisantes. Lorsque l'objectif est strictement pédagogique 
- celui de l'apprentissage d'une langue, d'une technique ou d'une formation - ou plutôt pratique, comme dans le cas de la traduction professionnelle, les critères varieront parfois considérablement. Ils sont fonction de la définition que l'on donnera de la traduction et du degré d'équivalence que l'on accordera au texte traduit par rapport à l'original.

De nombreuses méthodes proposent des formules d'évaluation des textes. Toutes partent de l'évaluation (ou du contrôle) de la qualité de la traduction à partir :

$1^{\circ}$ de la fidélité au message du TD (le fond);

$2^{\circ}$ de la forme sous laquelle ce message est exprimé.

Tels sont les deux grands critères fondamentaux qui ont engendré les principales méthodes utilisées pour juger de la qualité d'une traduction ${ }^{30}$. Selon le cas - traduction à caractère pédagogique, esthétique ou communicatif («pragmatique») _-, ils se subdivisent en un certain nombre de sous-ensembles adaptés à une situation de traduction particulière, et leur nombre varie en conséquence. Le Canada, par exemple, s'est illustré sur ce plan en établissant diverses formules de contrôle de la qualité des travaux de traduction. C'est ainsi que le Conseil des traducteurs et interprètes du Canada (CTIC) utilise, pour la correction de l'examen d'agrément que doivent présenter les membres des sociétés affiliées de traducteurs pour faire reconnaittre leur compétence professionnelle, un barème particulier qui reflète assez bien les pratiques en usage dans les écoles de traduction, les organisations internationales et le secteur privé.

\section{LE SICAL DU GOUVERNEMENT DU CANADA}

Pour sa part, le Bureau de la traduction du gouvernement du Canada (alors Bureau des traductions) a mis au point, en 1977, un Système canadien d'appréciation de la qualité linguistique (SICAL), composé au départ de 15 paramètres divisés en trois groupes. Depuis, ce système a été modifié à plusieurs reprises et simplifié. Il tend de plus en plus vers la formule minimale de l'évaluation du fond et de la forme, toujours à partir de critères de respect du sens de TD et de la forme imprimée au TA, qui doit correspondre aux normes et codes linguistiques en usage. Le réviseur, lorsqu'il évalue une traduction ${ }^{31}$, souligne la partie contestable et la qualité d'un simple $\mathrm{T}$ (pour traduction) ou $\mathrm{L}$ (pour langue), quand la faute relevée est sans gravité, ou, en cas de faute grave, de la même lettre, mais encerclée. Le texte est ensuite jugé acceptable ou non selon le nombre et la gravité des fautes relevées. Ce système relativement simple semble efficace, quoiqu'il convienne à un type de traduction bien particulier, celle qui caractérise un grand État institutionnellement bilingue comme l'est le Canada.

Plus en détail, le SICAL, qui est conçu pour évaluer les textes traduits et destinés à assurer le fonctionnement des rouages de l'État canadien, se fonde sur une conception particulière des normes de la traduction dans l'administration fédérale. Selon le
Guide de l'entrepreneur - Travaux de traduction ${ }^{32}$, pour être acceptable, une traduction doit «rendre le message du texte de départ avec exactitude, en une langue d'arrivée correcte, authentique et adaptée au sujet et à la destination» (p. 2). Chacun de ces quatre principes est ensuite expliqué dans le détail. Le but du SICAL consiste, pour le réviseur, à vérifier si les traductions sont bien conformes aux normes établies. Le texte, selon sa longueur, est révisé par tranches de $400 \operatorname{mots}^{33}$, et le résultat de l'évaluation est soit «satisfaisant», soit «non satisfaisant». Les textes sont divisés en cinq groupes alphabétiques (A-E), deux appartenant à la catégorie «satisfaisant» (A et B), et les trois autres à celle des «non satisfaisant» (C-E).

Il s'ensuit un nombre assez important de types de «fautes» (j'en ai relevé 16) affectant soit le transfert (du sens), soit la langue, donc les défauts d'expression. En tout, j'ai compté quelque 70 «fautes» possibles, de transfert et de langue ${ }^{34}$. C'est dire avec quelle minutie est évaluée la qualité des textes traduits.

Il s'agit, dans le cas du SICAL, de traductions professionnelles répondant à des critères de qualité précis, situés en aval dans le processus d'apprentissage. Du point de vue du formateur, l'évaluation d'une traduction, selon qu'elle portera sur des travaux d'étudiants du $1^{\text {er }}$ cycle ( $1^{\text {re }}, 2^{\mathrm{e}}$ et $3^{\mathrm{e}}$ années) ou des $2^{\mathrm{e}}$ et $3 \mathrm{e}$, vise un tout autre but, situé en amont: l'apprentissage de la traduction, soit former, corriger, perfectionner le futur traducteur, en vue de l'exercice probable de la traduction professionnelle. Aussi les formules d'évaluation seront-elles adaptées à l'objectif pédagogique visé, au type et à la longueur du texte utilisé, au domaine considéré, aux conditions d'apprentissage (traduction effectuée en classe, à la maison), etc. En conséquence, en pédagogie de la traduction et notamment dans les premières étapes de l'apprentissage, il est risqué de s'inspirer, sinon à titre occasionnel ou d'essai, de méthodes à vocation strictement professionnelle pour former les futurs traducteurs.

Le problème posé par l'évaluation d'une traduction débouche sur celui de la pratique de la traduction dans le processus d'apprentissage.

\section{PRATIQUE DE LA TRADUCTION}

C'est un truisme que d'affirmer que l'on ne devient traducteur qu'en traduisant. Toute activité exigeant un savoir-faire, une technique passe par une pratique intensive, généralement quotidienne. On connaît l'exemple des instrumentistes (pianistes, violonistes ou autres) qui consacrent chaque jour un certain temps à jouer de leur instrument, à faire des gammes, des exercices, à répéter leurs partitions. Et pourtant, il s'agit parfois de grands musiciens, de concertistes réputés. Le traducteur, tel le musicien, doit s'entraîner en vue de pratiquer son futur métier, et de préférence dans des conditions se rapprochant le plus possible de la situation réelle du traducteur professionnel, que ce dernier soit traducteur d'entreprise, 
fonctionnaire (dans la fonction publique locale, régionale ou nationale).

La question semblerait aller de soi. Or, il n'en va pas nécessairement ainsi et il existe encore trop de centres de formation de traducteurs dans le monde qui ne mettent pas l'accent sur l'acquisition d'une compétence professionnelle. Il s'ensuit un décalage parfois important, à l'issue des trois ou quatre années d'apprentissage, entre la formation reçue et les besoins, le niveau réel de la profession. Trop rares sont encore les pays où l'on est parvenu à établir un (relatif) équilibre entre les critères d'excellence fixés par les universitaires et les exigences dictées par les lois du marché national ou international. À l'ère des communications, du libre-échange et des marchés communs, les besoins se sont développés, et les niveaux, élevés. À défaut de le précéder, la formation doit suivre le mouvement.

Aussi la réflexion et l'activité pédagogiques doivent-elles concevoir et mettre en ouvre des formules d'apprentissage adaptées à ces nouveaux besoins, notamment sous forme de stage(s) offerts aux étudiants en cours, en fin de formation, voire à l'issue de celle-ci. Car tel est bien l'enjeu de ce complément indispensable de l'apprentissage des techniques de traduction. Non seulement cette activité est difficile à organiser (il faut au préalable s'assurer de la collaboration des entreprises, des services et des personnes concernés, dans le privé comme dans le public, ce qui ne va pas de soi), mais encore on doit en prévoir les modalités jusque dans le moindre détail, dont le moment, la durée, la nature de la supervision envisagée, les objectifs visés, l'évaluation permanente du travail accompli et celle des résultats obtenus, etc.

La formule du stage, à elle seule, ne saurait suffire. Elle peut - et doit - être complétée par d'autres activités pratiques, à caractère professionnel. Ce peut être la mise sur pied de journées de réflexion commune (formateurs, employeurs et étudiants), des journées de traduction, de révision, de terminologie, de correction (d'examen professionnel, par exemple), ou encore de documentation et de recherches documentaires, de stages internes ou externes, pontuels ou thematiques, de visites de services (de traduction, de terminologie, de documentation...), etc. La formule du stage est extensible à souhait et peut prendre de nombreuses formes, de la plus simple (le mini-stage ponctuel d'une matinée) à la plus complexe (stage de longue durée à volets multiples, par rotation, déplacement entre services), d'un lieu à un autre. À l'évidence, cela pose des problèmes d'organisation, de coordination, d'animation, de disponibilité et de collaboration entre enseignants, traducteurs et employeurs. Toutefois, il n'y a rien d'insurmontable en la matière et les résultats en valent largement la peine ${ }^{35}$.

S'il est illusoire de croire que l'étudiant, au terme de ses études, aura acquis la compétence professionnelle requise d'un traducteur expérimenté, il n'est pas interdit de penser que les écoles universitaires de traducteurs arriveront à former un professionnel «semi- fini» que le marché transformera selon les multiples besoins qu'il manifeste, lesquels, compte tenu de la diversité actuelle du monde des affaires, tendent vers l'infini. Aussi l'université ne peut-elle répondre à toutes les demandes particulières de formation que réclament les entreprises. Elle visera plutôt à dispenser une formation suffisamment générale et souple pour que le traducteur qui en sortira puisse s'adapter aux situations particulières qui se présenteront à lui et aux conditions d'exercice d'une profession exigeante.

Je crois que c'est à partir de ces axes fondamentaux que doivent s'orienter les réflexions méthodologiques en didactique de la traduction.

JEAN-Claude GÉmar Université de Montréal, Montréal, Canada

\section{Notes}

1. «Peut-on enseigner la traduction ?», Journal des traducteurs, vol. II, $n^{\circ} 4$, Montréal, p. 141.

2. Extrait d'une communication intitulée «De la conception à l'enseignement de la traduction», Actes du congrès de l'UQAM (23-31 mai 1980) sur $\mathrm{La}$ traduction: l' universitaire et le praticien, Éditions de l'Université d'Ottawa, 1984, p. 271.

3. Ibidem, p. 271

4. Selon l'expression définie par Sélim Abou en ces termes: «Nous entendons par groupe ethnique un groupe dont les membres possèdent, à leurs propres yeux et aux yeux des autres, une identité enracinée dans la conscience d'une histoire ou d'une origine commune». Extrait de «L'identité culturelle», Paris, Anthropos, p. 32. Cité par J.-C. Corbeil dans la préface à la $2^{2}$ édition de l'Introduction à la terminologie, de Guy Rondeau, Chicoutimi, Gaétan Morin Éditeur, 1984, p. xxiii.

5. À ce propos, un linguiste québécois, Jean-Claude Corbeil, dans une entrevue télévisée (aux nouvelles de Radio-Canada, le 3 avril 1992), parle de «l'urticaire linguistique» dont sont régulièrement frappés les pouvoirs publics dès lors qu'il est question de langue. On constate cette réaction dans de nombreux pays, et non exclusivement au Canada.

6. Ce qui sous-entendrait que l'on a pris en compte un nombre très élevé de variables, dont, entre tant d'autres, le niveau (1 $1^{\mathrm{er}}, 2^{\mathrm{e}}$ ou $3^{\mathrm{e}}$ cycles) des études considérées, leur durée et les objectifs d'apprentissage visés, les débouchés envisagés, le niveau des étudiants (diplômés du $1^{\text {er }}$ cycle ou non), leurs connaissances linguistiques, etc.

7. L'auteur de ces lignes a présenté un rapport sur la pédagogie de la traduction lors du congrès consacré à «La traduction: l'universitaire et le praticien», déjà cité, rapport figurant dans les actes de ce congrès, aux pages 281-282 (cf. note 3 , supra).

8. Traduire, dossier composé sous la direction de J.-C. Gémar pour les participants à la Quatrième rencontre mondiale des départements d'études 
françaises, Jawaharlal Nehru University, New Delhi (15-19 déc. 1988), AUPELF, 66 p.

9. Rappelons-les pour mémoire, dans l'ordre de difficulté croissante énoncé par les auteurs : emprunt, calque, traduction littérale, transposition, modulation, équivalence, adaptation (édition internationale de Didier, 1971, p. 55).

10. Jean Darbelnet lui-même nous a assuré, à de nombreuses reprises, que les mots «méthodes de traduction» qui figurent sur l'édition originale du livre avaient été ajoutés par l'éditeur, qui craignait que le titre retenu ne décourage d'éventuels utilisateurs ou ne les induise en erreur!

11. «Autrement dire... Pour une redéfinition des stratégies de formation des trađucteurs", Meta, déc. 1991 , pp. 543 et suiv.

12. Ibidem, p. 545

13. Dont celle du remarquable travail accompli par Jean Delisle, auteur de L'analyse du discours comme méthode de traduction, op.cit.

1.4. Si tant est que l'on puisse cerner avec précision la nature de cette «réalité». En effet, chaque entreprise (ou service) de traduction a tendance à croire que la terre tourne autour de l'axe qu'elle constitue et donc à voir dans ses besoins particuliers ceux de la collectivité tout entière, alors qu'elle ne représente que le plus commun dénominateur (particulier) du plus grand commun dénominateur : la société dans son ensemble.

15. Et cette remarque ne vise pas uniquement les pays en développement; des pays développés, plus nombreux qu'on ne le croit généralement, pratiquent cncore avec excès cette forme d'enseignement détaché de la réalité.

16. Comme nous le faisions remarquer, en 1980 , déjà, aux participants aux SEDIFRALE II (San José de Costa Rica, 4-8 février 1980) dans une communication présentée en congrès sur le thème de la formation du traducteur.

17. C'est ce qui ressort de l'étude de Beatriz Geller, Les traducteurs de documentation en France..., déjà citée, où l'on apprend qu'une partie importante, qui pourrait atteindre les $50 \%$, des traducteurs professionnels n'ont pas fait d'études de traduction, voire ne possèdent pas de diplôme universitaire (p. 53). Nous rappelons qu'il s'agit de la France, pays doté d'une solide et ancienne tradition universitaire. On imagine aisément quelle peut (ou doit) être la situation dans les pays moins favorisés.

18. Dont, entre autres, le droit, l'histoire, la philosophie, les langues mortes.

19. Illustrons cette affirmation par quelques exemples relevés dans des copies d'étudiants en traduction: 1. [...] that language had pretty well died of its own accord. cette langue était belle ( $\mathrm{sic}$ ) et bien morte.

2. In the majority opinion, Justice Potter Steward observed..

Selon la majorité des gens, Steward, le potier (sic !) de la Justice...
3. [...] at a school near the arrest site. une école à côté de l'arrêt de l'autobus.

Exemples où l'on voit que si, parfois, les connaissances linguistiques font défaut pour comprendre parfaitement le TD, les lacunes peuvent être tout à la fois linguistiques, au départ (LD) comme à l'arrivée (LA), et culturelles ( $c f .1$ et 2 ).

20. À commencer par le premier d'entre eux: l'État canadien, dont le Bureau de la traduction dénonce régulièrement cette lacune, constatée chez les diplômés des écoles de traduction.

21. À l'ESIT, par exemple, les candidats doivent posséder un diplôme de $1^{\text {er }}$ cycle pour présenter leur candidature. À l'Université de Montréal, les étudiants entreprennent leurs études au 1 er cycle. Les détenteurs d'un diplôme de $1^{\mathrm{er}}$ cycle, toutefois, peuvent entreprendre des études au $2^{\mathrm{e}}$ cycle (Maitrise en traduction) après avoir suivi une année préparatoire à la maîtrise.

22. La querelle, en la matière, est aussi virulente que celle des Anciens et des Modernes. Il pourrait bien s'agir, d'ailleurs, de la même chose: pour les uns (nostalgiques du passé), le niveau des connaissances ne cesserait de baisser (en sorte que, comme dit l'autre, depuis qu'il baisse, c'est-à-dire depuis toujours, il devrait avoir atteint le degré 0 ! Pour les autres, les optimistes, au contraire, le niveau général des connaissances des jeunes générations n'a cessé de s'élever. En fait, le problème de la langue n'est que le révélateur d'un changement radical d'attitude de la société envers ce qui coûte, demande un effort. Contrairement à ce que l'on pense, l'apprentissage de sa propre langue est tout aussi difficile que celui des sciences ou d'une langue étrangère. Voir sur la question de la "crise des langues», par exemple les dossiers suivants: «Le français miroir des Français», L'Express, 4 avril 1986, pp. 58-64, où les auteurs nous font remarquer que, de nos jours, «parler comme un livre» ne correspond plus à la réalité, alors que les gens écriraient plutôt comme ils parlent...; un livre traite la question en détail : La crise des langues, Conseil de la langue française, Éditeur officiel du Québec et Éditions Le Robert, 1985. Quel que soit son objet, la question passionne, notamment les francophones. En témoigne le débat virulent qui a récemment opposé partisans et adversaires de la réforme de l'orthographe de la langue française, pas moins animé que celui qui a enflammé les lusophones, au Portugal et au Brésil, lors de la réforme du portugais.

23. Exemples authentiques extraits de notre recueil personnel de fautes «mémorables».

24. La consultation du célèbre ouvrage de Maurice Grevisse, Le Bon Usage, en est une preuve dirimante: il est bien difficile de se prononcer péremptoirement sur cette question de norme, les bons auteurs différant souvent d'avis entre eux, comme le prouvent les exemples relevés par l'auteur. 
25. Ces problèmes ont été énoncés dans l'introduction à plusieurs ouvrages de référence que nous avons publiés, seul ou en collaboration, notamment les bibliographies suivantes: Bibliographie sélective du traducteur - Commerce et économie, Montréal, Linguatech, 1977, 180 p. + addendum (1979), 30 p.; Bibliographie sélective du traducteur - Droit et justice, Montréal, Linguatech, 1978,280 p.; Les outils du traducteur. Bibliographie sélective et critique, Montréal, AUPELF, 1987, 377 p. + LIV p. [franç., esp., port.].

26. Nous pensons notamment aux 22 volumes de la dernière édition de l'Oxford English Dictionary (OED) et aux 16 du Trésor de la langue française (TLF).

27. Signalons au passage l'entreprise hors du commun du Dictionnaire explicatif et combinatoire du sémanticien Igor Mel'c-- k, qui tend vers l'exhaustivité des sens possibles des mots, par les fonctions qu'ils occupent dans le discours.

28. Albert Jacquard, le célèbre généticien, s'est abondamment exprimé sur ce sujet. Ses travaux sur l'intelligence, la «valeur» et l'évaluation font autorité. Voir, en particulier, deux de ses ouvrages sur la question: Éloge de la différence, Paris, Seuil, 1978, et Au péril de la science, Paris, Seuil, 1982. Une thèse de doctorat sur le processus de compréhension en traduction a été soutenue en 1990 à l'Université de Montréal par Jeanne Dancette (Étude réflexive et expérimentale du processus de compréhension dans l'activité de tra- duction, Dêp. de linguistique et philologie, Faculté des arts et des sciences). On ne saurait en effet parler d'évaluation sans aborder la question de la «compréhension» et de sa vérification.

29. Voir sur cette question le livre de Paul Horguelin (1978) : Pratique de la révision, Montréal, Linguatech, qui traite le sujet sous tous ses aspects, notamment historiques.

30. Cette question a fait l'objet d'une thèse de doctorat soutenue à l'Université de Montréal en 1985 par Robert Larose: Paramètres d'évaluation des traductions. Théories contemporaines et approche textologique.

31. En fait, une partie de la traduction, soit trois fois 400 mots dans un texte donné.

32. Bureau des traductions, Secrétariat d'État, 1984

33. Nombre arbitrairement fixé à cette hauteur, mais correspondant grosso modo à une page de texte, soit, selon la norme des Nations unies, 1 TPU (Translation Page Unit).

34. Dans le livre Aide-mémoire d'autoperfectionnement à l'intention des traducteurs et des rédacteurs établi par le Secrétariat d'État du Canada, $1987,230 \mathrm{p}$.

35. Organisateur nous-même de stages internationaux de formation, de perfectionnement et d'orientation depuis de nombreuses années, nous avons éprouvé la plupart de ces difficultés et les avons généralement surmontées. 